\title{
Suppression of superconductivity by non-magnetic disorder in the organic superconductor $\left(\mathrm{TMTSF}_{2}\left(\mathrm{ClO}_{4}\right)_{(1-x)}\left(\mathrm{ReO}_{4}\right)_{x}\right.$
}

\author{
N.Joo ${ }^{1,2}$, P.Auban-Senzier ${ }^{1}$, C.R.Pasquier ${ }^{1}$, P.Monod ${ }^{3}$, D.Jérome ${ }^{1}$ and K.Bechgaard ${ }^{4}$ \\ ${ }^{1}$ Laboratoire de Physique des Solides (CNRS,UMR 8502), Université Paris-Sud, 91405 Orsay, France \\ ${ }^{2}$ Faculté des Sciences de Tunis, LPMC, Campus Universitaire, 1060 Tunis, Tunisie \\ ${ }^{3}$ Laboratoire de Physique des Solides (CNRS,UPR 5 ), ESPCI, 75231 Paris-Cedex 05, France \\ ${ }^{4}$ Polymer Department, Risø National Laboratory, 4000 Roskilde, Denmark
}

\begin{abstract}
We present a study of the superconducting properties $\left(T_{c}\right.$ and $\left.H_{c 2}\right)$ in the solid solution $\left(\mathrm{TMTSF}_{2}\left(\mathrm{ClO}_{4}\right)_{(1-x)}\left(\mathrm{ReO}_{4}\right)_{x}\right.$ with a $\mathrm{ReO}_{4}^{-}$nominal concentration up to $x=6 \%$. The dramatic suppression of $T_{c}$ when the residual resistivity is increased upon alloying with no modification of the Fermi surface is the signature of non-conventional superconductivity . This behaviour strongly supports $p$ or $d$ wave pairing in quasi one dimensional organic superconductors. The determination of the electron lifetime in the normal state at low temperature confirms that a single particle Drude model is unable to explain the temperature dependence of the conductivity and that a very narrow zero frequency mode must be taken into account for the interpretation of the transport properties.
\end{abstract}

Organic superconductivity has been around for nearly 25 years since its discovery in $(T M T S F)_{2} P F_{6}[1]$. However very little is still known experimentally regarding the symmetry of the superconducting pairing. An early theoretical work of Abrikosov [2] performed within the self consistent approximation has concluded that both scenarios could be envisaged for quasi one dimensional (Q-1D) conductors namely, singlet or triplet spin pairing depending on the properties of the electron-electron interaction. It was also anticipated that the stability of the triplet state would be very sensitive to the presence of non magnetic impurities.

An other approach based on the exchange of antiferromagnetic fluctuations between neighbouring chains has led to an exotic $d$-like pairing 3 . This model is singlet in the spin sector and leads to a superconducting gap which exhibits a sign reversal along the open Fermi surface of these Q-1D conductors. Experiments available up today are unable to settle without ambiguity the question of the pairing symmetry in organic conductors. As far as one dimensional superconductors are concerned, some support in favour of triplet pairing was claimed from the analysis of critical fields in $(T M T S F)_{2} \mathrm{ClO}_{4}$ and $(T M T S F)_{2} P F_{6}$ in terms of pure type II superconductivity [4] and also from the observation of a divergence of the upper critical field at low temperature for fields parallel to the intermediate direction $\mathrm{b}^{\prime}$ with $H_{c 2}(T)$ exceeding greatly the paramagnetic (Pauli) limit value in $(T M T S F)_{2} P F_{6}[\underline{5}$. In addition, the spin susceptibility found to be temperature independent in the superconducting phase and equal to its normal state value for $(T M T S F)_{2} P F_{6}$ has been taken as an other evidence in favour of triplet pairing [6]. However, both experiments may not be fully convincing since in the former the critical field has been investigated in a pressure domain close to the low pressure spin density wave instability where superconductivity is inhomogenous [7, 8] whereas for the latter it is unclear whether the NMR signal needed for the Knight shift measurement refers to superconducting or to normal electrons in this superconducting state exhibiting a vortex structure. For $(\mathrm{TMTSF})_{2} \mathrm{ClO}_{4}$, another member of the Bechgaard salts in which superconductivity is stable under ambient pressure [9] the situation remains also unclear. The finding of a power law dependence of the relaxation rate in the superconducting phase down to $T_{c} / 2$ led Takigawa et-al [10] to suggest the existence of lines of zeros for the superconducting gap on the Fermi surface 11] while thermal conductivity data down to $T_{c} / 7$ provided compelling evidences for a nodeless gap [12]. As far as the 2-D organic superconductors built on the BEDT - TTF molecule are concerned the actual symmetry of the superconducting wave function is still controversial although far more experimental studies have been conducted. Most NMR investigations support a spin-singlet pairing 13, 14, 15. Recent specific heat measurements performed in two members of the $B E D T-T T F$ family have shown a fully gapped order parameter [16, 17] at variance with early heat conduction 18] and penetration depth measurements 19] providing evidences for nodes in the gap. This contradictory situation might be reasonably resolved by recent penetration depth experiments pointing out the critical influence of the ethylene groups ordering on the existence of low energy electron excitations 20]. We may notice that $T_{c}$ in these 2-D superconductors is very sensitive to the existence of intrinsically non-magnetic disorder 21].

The quantum states of the partners entering into the formation of a Cooper pair of a BCS $s$-wave superconductor are related to each other by a time reversal symmetry. Hence no pair breaking is expected from the scattering of electrons against spinless impurities 22. Experimentally, this property has been verified in non-magnetic 
dilute alloys of $s$-wave superconductors and brought a strong support for the BCS model of conventional $s$-wave superconductors. However, the condition for time reversal symmetry is no longer met for the case of $p$-wave pairing and consequently $T_{c}$ for these superconductors should be strongly affected by even a small amount of such nonmagnetic scattering. It is the extreme dependence of the critical temperature of $\mathrm{Sr}_{2} \mathrm{RuO}_{4} \quad$ 23] on non-magnetic disorder which has provided a strong support in favour of triplet superconductivity in this compound. It is also the remarkable sensisitivity of organic superconductivity to irradiation [24, 25] which led Abrikosov to suggest the possibility of triplet pairing in these materials 2. Although irradiation was recognized to be an excellent method for the introduction of defects in a controlled way 26], defects thus created can be magnetic 27] and the suppression of superconductivity by irradiation induced defects as a signature of non-conventional pairing must be taken with "a grain of salt" since local moments can also act as strong pair-breakers on $s$-wave superconductors.

Using chemistry to break the crystalline invariance is an other way for the creation of local non-magnetic scatterers. This has been achieved by the substitution of TMTSF for TMTTF molecules in $T M_{2} X$ salts with $X=P_{6}[28]$ and $X=\mathrm{ClO}_{4}[29]$. However in both situations cationic alloying involves drastic modification of the normal state electronic properties since the SDW transition of (TMTSF) ${ }_{2} P F_{6}$ is quickly broadened and pushed towards higher temperature upon alloying 30. Consequently, such a cationic alloying may not be the best case to test the robustness of superconductivity against an enhanced scattering. A softer way of introducing disorder on the cation stack has been developed with the series (trimethyl-TS $\left.F_{(1-x)} T M T S F_{x}\right)_{2} X$ where a drastic suppression of superconductivity of $\mathrm{X}=\mathrm{ClO}_{4}$ is observed with $10 \%$ alloying while the SDW instability of $X=P F_{6}$ which involves an order of magnitude higher in energy is left unperturbed 29].

Leaving the cation stack uniform, scattering centers can also be created on the anion stacks. The role of the anion stack on the ground state is enhanced as soon as the anion which is located at an inversion center of the structure does not possess a central symmetry 31 . This is the case in particular for tetrahedral anions such as $X=\mathrm{ClO}_{4}$ which order at low temperature $\left(T_{A O}=24 \mathrm{~K}\right)$ in line with entropy minimization. As anion reorientation requires a tunneling process between two states at equal energy but separated by a large potential barrier, the dynamics of orientation is a slow process at low temperature. Hence, for samples slowly cooled through $T_{A O}$ (in the so-called R-state) the orientation of the anions is uniform along the stacking axis but alternate along the b-direction leading in-turn to a doubled periodicity with a concomitant opening of an energy gap on the Fermi surface at $\pm \pi / 2 b$ and the creation of two sheets of open Fermi surfaces at $+\mathrm{k}_{F}$ and $-\mathrm{k}_{F}$ respectively. Fast cooled samples reach low temperature in a state (the Qstate) where orientational disorder is frozen-in (i.e. a mixture of both anion orientations) and superconductivity is depressed 32, 33]. When the cooling rate is fast enough (quenched state) full disorder is preserved and the single-sheet Fermi surface of the high temperature sructure prevails at low temperature leading in turn to the instability of the metallic phase against a SDW ground state at $T_{S D W}=5 \mathrm{~K} 32$. Furthermore, it has been shown that neither the Pauli susceptibility 32, 34] nor the density of states 33] of the normal phase are affected by the orientational disorder introduced by the fast cooling procedure as long as a superconducting ground state is observed. An other approach for the introduction of anionic disorder has been successful with the synthesis of an anionic solid solution involving anions of similar symmetry. For centrosymmetrical anions a suppression of the SDW state has been observed in $(T M T S F)_{2}\left(A s F_{6}\right)_{(1-x)}\left(S b F_{6}\right)_{x}$ [35] but the effect on superconductivity which would have required a high pressure has not been studied in details. As far as the noncentrosymmetrical anions are concerned, the early studies by Tomić et-al [36] in $\left(\mathrm{TMTSF}_{2}\left(\mathrm{ClO}_{4}\right)_{(1-x)}\left(\mathrm{ReO}_{4}\right)_{x}\right.$ have shown that both the low temperature conductivity and the transition towards superconductivity are very strongly affected by alloying although X-ray investigations have revealed that long range order is preserved up to $3 \% \mathrm{ReO}_{4}^{-}$with a correlation length $\xi_{a}>200 \AA$ 37].

In the present paper we have investigated the influence of non-magnetic disorder on the superconducting properties of the solid solution $\left.(\mathrm{TMTSF})_{2}\left(\mathrm{ClO}_{4}\right)_{(1-x)}(\mathrm{ReO})_{4}\right)_{x}$ with different cooling rates down to $100 \mathrm{mK}$. ¿From the measurement of $T_{c}$ and of the upper critical field at different $x$ and cooling rates we have derived the relation between the coherence length and the critical temperature which is at variance with the expectation in a conventional dirty type II superconductor.

Single crystals of the solid solution $\left(\mathrm{TMTSF}_{2}\left(\mathrm{ClO}_{4}\right)_{(1-x)}\left(\mathrm{ReO}_{4}\right)_{x}\right.$ with $x$ in the range $0 \leq x \leq 0.06$ have been prepared with the usual electrocrystallization technique. As the relative concentration of $\mathrm{ReO}_{4}^{-}$and $\mathrm{ClO}_{4}^{-}$anions in the crystals could differ from the nominal concentration of the solution a microprobe analysis has been attempted. However the sensitivity of such a technique with respect to the $\mathrm{ReO}_{4}^{-}$ concentration is rather limited [38]. Only the $x=0.06$ sample gave a rhenium peak with a signal to noise ratio which was large enough to enable a calibration. We found that the actual concentration is about $30 \%$ smaller than the value expected from the nominal concentration with a large error bar. We shall see in the following that the nominal concentration is not so relevant for the purpose of our experiment.

Conductivity measurements have been conducted us- 


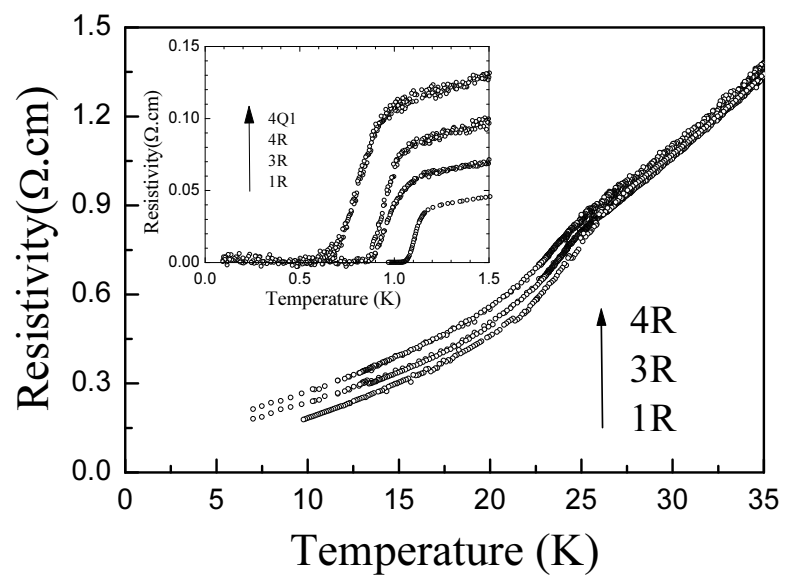

FIG. 1: Temperature dependence of the resistivity in the low temperature domain for three typical $\left(\mathrm{TMTSF}_{2}\left(\mathrm{ClO}_{4}\right)_{(1-x)}\left(\mathrm{ReO}_{4}\right)_{x}\right.$ samples with different $\mathrm{ReO}_{4}^{-}$concentrations in the relaxed state. The transition towards superconductivity is displayed in the insert for non-alloyed (1) and alloyed (3 and 4) samples in relaxed (R) or quenched $(\mathrm{Q})$ states $(\mathrm{Q} 1$ corresponds to the cooling rate of $-4 K / m n)$.

ing a low frequency lock-in detection with the current flowing along the $c^{\star}$ axis of the crystals. This direction has been chosen for transport studies because it allows a reliable cooling without visible cracks and also because the $a-b^{\prime}$ plane due to the high transport anisotropy provides a well defined equipotential surface for the resistance measurements . Contacts were made with silver paste on two gold pads evaporated on each parallel side of crystals with typical sizes $1 \times 0.3 \times 0.15 \mathrm{~mm}^{3}$. The low temperature was provided by a dilution refrigerator designed for convenient operation between $100 \mathrm{mK}$ and $30 \mathrm{~K}$ with a sample holder able to measure two samples simultaneously. For the comparison of the low temperature resistivity between different samples which is needed in this work we did not rely on their geometrical factors relating resistance to resistivity. This factor is always difficult to determine with much accuracy due to uncertainties in the measurement of the sample dimensions. Instead, we chose $28 \Omega \mathrm{cm}$ for the value of the room temperature transverse resistivity $\rho_{c^{\star}}$, an average derived from the study of 15 different samples. Subsequently we have normalized all measurements of resistance versus temperature against this average resistivity. This procedure is justified by the application of the Mathiessen's law which is particularly relevant in this experiment since the resistance ratio between ambient and helium temperature even in the most impure samples was at least 100. The ESR linewidth data displayed in fig. (2) bring a further justification for the procedure used to compare resistivity between different samples. Furthemore, we chose to characterize the electronic life time at low temperature by the residual resistivity $\rho_{0}$ which we obtain as the constant parameter in a polynomial fit of the temperature dependence of the resistivity in the normal state (instead of the nominal concentration of the solid solution). The resistivity is linear (quadratic) in temperature below (above) $10 \mathrm{~K}[39]$.

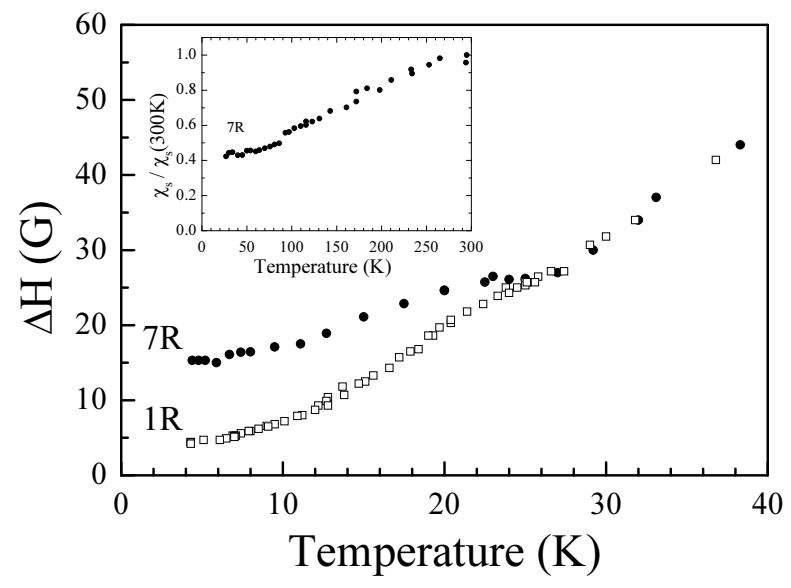

FIG. 2: ESR linewidth of pure (1R) and doped (7R) samples. The difference in temperature dependences of the two samples below $T_{A O}$ is due to the enhancement of the electronic scattering rate in the doped system. The anion ordering is somewhat smeared by a small temperature lag $(\approx 2 \mathrm{~K})$ existing between the thermometer and the sample in the range $24 \mathrm{~K}-12 \mathrm{~K}$. The inset shows the ESR susceptibility of the doped sample normalized at $300 \mathrm{~K}$. The temperature dependence is in close agreement with that of $(\mathrm{TMTSF})_{2} \mathrm{ClO}_{4}$ published in the literature.

Reliable data require a good control of the anion ordering, therefore the procedure for low temperature measurements was the following: the sample was first slowly cooled down to $4.2 \mathrm{~K}(\leq 0.2 \mathrm{~K} / \mathrm{mn})$, then warmed up to $30 \mathrm{~K}$ and subsequently cooled again to low temperature with an adequate control of the cooling rate through the anion ordering temperature. The cooling rate used to stabilize the R-state of $(T M T S F)_{2}\left(C l O_{4}\right)_{(1-x)}\left(\mathrm{ReO}_{4}\right)_{x}$ in our experiments is $-0.05 \mathrm{~K} / \mathrm{mn}(3 \mathrm{~K} / \mathrm{h})$. This rate is indeed much lower than the lowest rate used earlier to study the R-state (12 and $5 \mathrm{~K} / \mathrm{h})$ 36]. Figure (1) displays resistivity data in the low temperature domain. In the present study we have focused our attention on the samples with $0 \leq x \leq 0.06$ measured with cooling rates slow enough in order to preserve a metallic behaviour $(\delta \rho / \delta T>0)$ over the entire temperature regime. According to fig (1) the signature of anion ordering is visible at $T_{A O}=24 \mathrm{~K}$ and survives although smeared out upon alloying up to the nominal concentration of $x=0.06$. No decrease of $T_{A O}$ larger than $1 \mathrm{~K}$ or so has been noticed in agreement with X-ray investigations 37, 40, 41]. In addition we notice that the residual resistivity increases with the concentration of $\mathrm{ReO}_{4}^{-}$defects in the $\mathrm{R}$-phase and also with the cooling rate for a given sample in agreement with Mathiessen's law, see insert in fig.(11). We also noticed that much lower cooling rates are necessary to reach a $\mathrm{R}$ 
state in highly defective samples in agreement with the increase of the annealing time requested by annealing close to $T_{A O}$ as measured by Tomić 32.

Electron paramagnetic resonance studies have been carried on in a X-band spectrometer equipped with a variable temperature cryostat able to control the cooling rate down to $4.2 \mathrm{~K}$. Typical results are shown on fig (2) where the peak to peak linewidths $(\Delta \mathrm{H})$ of pure $(1 \mathrm{R})$ and $x=0.06(7 \mathrm{R})$ samples are displayed against temperature. Figure (2) reveals a temperature dependence as expected for the resonance of a conduction electron spin resonance (CESR) in metals, namely $\Delta \mathrm{H} \propto$ electronic scattering rate (resistivity) 42]. The magnetic field is along the $b$ axis of the single crystals and the g factor has been found temperature independent. The CESR data show that Mathiessen's law applies fairly well to $\Delta \mathrm{H}$ since similar increases of linewidth and resistivity are observed upon doping. Furthermore, $\mathrm{ReO}_{4}$ doping has no effect on the integrated electron susceptibility temperature dependence as it should if the doped samples are free from magnetic impurities, see inset.

For all samples the superconducting transition can be detected by a drop of the resistance to zero. The onset temperature was defined by the intercept between the high temperature extrapolation and the linear fit in the transition region. In the pure sample it amounts to $1.18 \mathrm{~K}$ with a $(10 \%-90 \%)$ width of $0.1 \mathrm{~K}$.

When a magnetic field is applied along the $c^{\star}$ direction, the whole $R(T)$ curve is pushed towards low temperature. Hence $H_{c 2}(T)$ can be derived for different samples and different cooling rates, see fig (3). According to the data of fig (3) the temperature dependence of $H_{c 2}(T)$ is linear close to $T_{c}$ as expected from the Ginzburg-Landau theory 43. and a value for $T_{c}$ can thus be determined by the zero field extrapolation of the critical field [44].

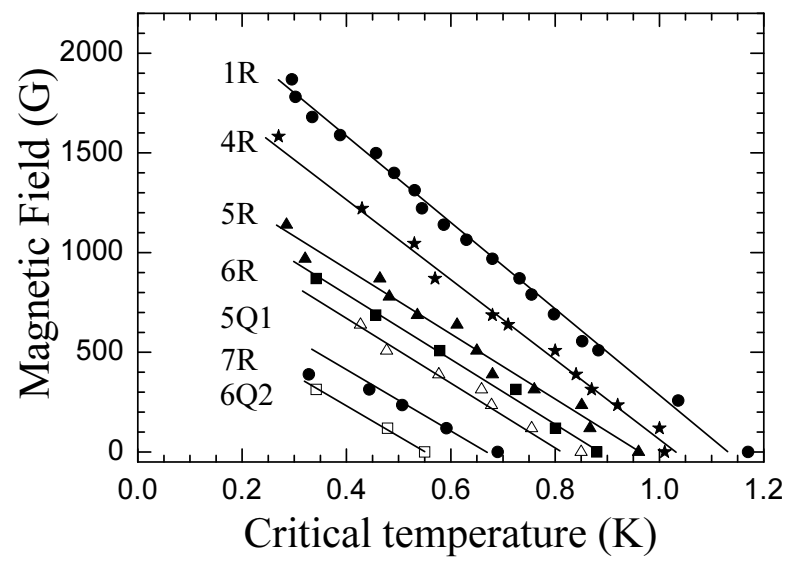

FIG. 3: Critical temperature vs magnetic field for five crystals of $\left(\mathrm{TMTSF}_{2}\left(\mathrm{ClO}_{4}\right)_{(1-x)}\left(\mathrm{ReO}_{4}\right)_{x}\right.$ with different $T_{c}, \mathrm{Q} 1$ and Q2 refer to quenched samples with cooling rates of $-4 K / m n$ and $-26 K / m n$ respectively.

The central result of this work is shown on fig (4) where

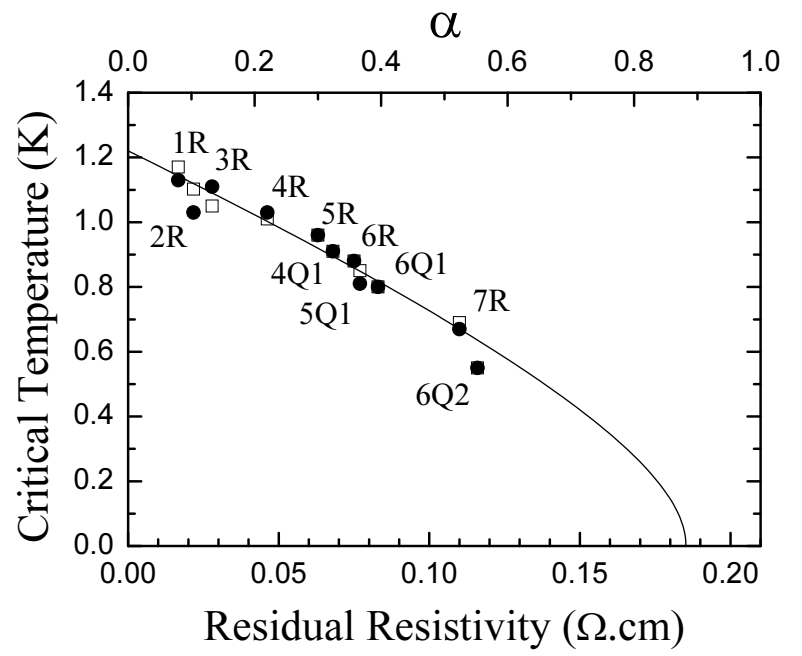

FIG. 4: Superconducting critical temperature derived from the zero field intercept of $H_{c 2}(T)$ in fig (3) (dots) and also from the zero field resistivity data (squares) as a function of the residual resistivity for samples with different amounts of disorder either chemical or(and) orientational. The solid line is a least square fit of the digamma pair-breaking function to the data which reaches zero at $\alpha=0.88$.

$T_{c}$ is plotted against the residual resistivity in the transverse direction. On this figure the data which are displayed come from different samples in the $\mathrm{R}$ state and also from quenched samples preserving the metallic behaviour and superconductivity at low temperature. It can be inferred that the residual resistivity is proportional to the inverse electron life time $1 / \tau$ (or inverse mean free path $1 / l)$. This is the case for coherent transport along $c^{\star}$ [4] but also for incoherent transport since then we also have $\rho_{0 c \star} \propto 1 / \tau$ [46, 47]]. The relation between the transverse resistivity and the in-chain inverse electron lifetime is still valid in case of incoherent transverse transport as shown recently by the perturbative derivation of the transverse conductivity in the tunnelling approximation 48, 49. On account of the small value of the overlap along the $c$ direction for $(\mathrm{TMTSF})_{2} \mathrm{ClO}_{4}$, the $c$-axis transport is probably at the border between coherence and incoherence. However, the observation of a narrow angular magnetoresistance peak when a large magnetic field is applied perpendicular to the $c$ axis supports the possibility of coherent transport at low temperature [50]. Figure (4) shows that the extra scattering due to orientational disorder in fast cooled samples adds to the residual scattering due to the chemical defects. From the signature of the anion ordering at $T_{A O}$ we can be confident that the density of states at the Fermi energy is not changed in the solid solution. In addition an effect of volume change can also be ruled out to explain the data on fig (4) since the substitution of $\mathrm{ReO}_{4}^{-}$to $\mathrm{ClO}_{4}^{-}$should expand the unit cell and in turn increase $T_{c}$ at variance with the data [51]. Consequently, 
the suppression of $T_{c}$ must be related to the enhancement of the scattering rate in the solid solution. The additional scattering cannot be ascribed to magnetic scattering according to our ESR studies. Thus, our results cannot be reconciled with the picture of a superconducting gap keeping a constant sign over the whole $\left( \pm k_{F}\right)$ Fermi surface and require a picture of pair breaking in a superconductor with an unconventional gap symmetry. The conventional pair breaking theory for magnetic impurities in usual superconductors can thus be generalized to the case of non-magnetic impurities in unconventional materials and $T_{c}$ reads 52 , 53],

$$
\ln \left(\frac{T_{c 0}}{T_{c}}\right)=\Psi\left(\frac{1}{2}+\frac{\alpha T_{c 0}}{2 \pi T_{c}}\right)-\Psi\left(\frac{1}{2}\right)
$$

with $\Psi$ being the digamma function, $\alpha=\hbar / 2 \tau k_{B} T_{c 0}$ the depairing parameter, $\tau$ the elastic scattering time and $T_{c 0}$ the limit of $T_{c}$ in the absence of any scattering. According to fig (4) we obtained $T_{c 0}=1.22 \mathrm{~K}$ from the best least square fit of the data. The critical scattering rate for the suppression of superconductivity leads to $1 / \tau_{c r}=$ $1.44 \mathrm{~cm}^{-1}, \tau_{c r}=3.5 \mathrm{ps}$ (following the definition of $\alpha$ ). Accordingly, $1 / \tau$ amounts to $0.13 \mathrm{~cm}^{-1}(\tau=40 \mathrm{ps})$ in the pristine $(\mathrm{TMTSF})_{2} \mathrm{ClO}_{4}$ sample.

It is now interesting to make the connection with the rate which can be derived from a Drude analysis of the longitudinal transport using the usual relation $\sigma_{D C}=\omega_{p}^{2} \tau / 4 \pi$ between transport and plasma frequency data. Given a resistance ratio of 800 between 300 and $2 \mathrm{~K}$ as currently measured in pure $(\mathrm{TMTSF})_{2} \mathrm{ClO}_{4}$ in the absence of cracks and a plasma frequency obtained from the room temperature optical data 54 the Drude mode should have a width at half height of $\approx 2 \mathrm{~cm}^{-1}$ at low temperature. This width is admittedly much larger than what has been derived from the depression of superconductivity by non-magnetic impurities (about 15 times larger). However, our finding of a very long scattering time in pure $(\mathrm{TMTSF})_{2} \mathrm{ClO}_{4}$ supports the claims made from polarized far-infrared spectroscopy data for a pseudo-gap with a low conductivity in the $2-4 \mathrm{~cm}^{-1}$ frequency range together with the existence of a zero frequency peak whose width is less than $0.034 \mathrm{~cm}^{-1}$ for light //a below 10K 55, 56]. The DC conductivity at low temperature would in turn correspond to the conductivity of this narrow mode at zero frequency.

The suppression of superconductivity at a critical value of the scattering rate corresponds to a critical mean free path of the order of the microscopic superconducting coherence length $\left(l \approx \xi_{0}\right)$. Since the coherence length is anisotropic in such a low dimensional conductor our data are unable to measure the different components. We have only access to its average $\xi_{a b}$ in the $a-b^{\prime}$ plane using the Ginzburg-Landau theory and $T_{c} d H_{c 2} / d T=\phi_{0} / 2 \pi \xi_{a b}^{2}$.

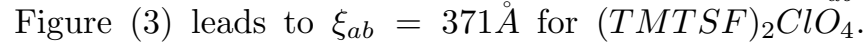
This value can be compared with a determination performed by Murata et-al [57] who obtained $\xi_{a b}=486 \AA$.
With fig (4), our determination of the coherence length gives $l \approx 4100 \AA\left(l / \xi_{0}=11\right)$ in $(T M T S F)_{2} \mathrm{ClO}_{4}$. Hence we can be confident that pristine $(\mathrm{TMTSF})_{2} \mathrm{ClO}_{4}$ is located in the clean limit and should be treated as a clean superconductor.

The data of fig (4) and fig (3) cannot be reconciled within the framework of a conventional superconductor. First, the slope $d H_{c 2} / d T$ shows a tendency to decrease together with $T_{c}$ which is the behaviour expected in a clean type II superconductor when $T_{c}$ is decreased but in such a situation $T_{c}$ itself should not be affected by non magnetic impurities. Second, in a dirty type II material one can expect $d H_{c 2} / d T \alpha \rho_{0}[43]$. This is opposite to the experimental behaviour in fig (3).

In summary, the solid solution $\left(\mathrm{TMTSF}_{2}\left(\mathrm{ClO}_{4}\right)_{(1-x)}\left(\mathrm{ReO}_{4}\right)_{x}\right.$ provides an ideal case for the study of the response of organic superconductivity to non-magnetic disorder. We have shown that on the dilute side with a nominal concentration of $\mathrm{ReO}_{4}$ up to $x=0.06$ the signature of the anion ordering at $24 \mathrm{~K}$ provides a proof for the preservation of the original Fermi surface whereas the superconducting transition temperature is depressed by a factor about two. This behaviour cannot be reconciled with a model of conventional superconductors. The gap must show regions of positive and negative signs on the Fermi surface which can be averaged out by a finite electron lifetime due to elastic scattering. As these defects are local the scattering momentum of order $2 k_{F}$ can mix + and $k_{F}$ states and therefore the sensitivity to non-magnetic scattering is still unable to tell the difference between $p$ or $d$ orbital symmetry for the superconducting wave function. Furthermore, the conclusions of our work corroborate a previous experimental conclusion showing that the sole existence of a gap anisotropy was unable to explain the concomitant decreases of both $T_{c}$ and specific heat anomaly in $(\mathrm{TMTSF})_{2} \mathrm{ClO}_{4}$ when the cooling rate is increased [58]. A noticeable progress could be achieved paying attention to the spin part of the wave function. In the close vicinity of $T_{c}$ orbital limitation for the critical field is expected to prevail and therefore the analysis of the critical fields close to $T_{c}[\underline{4}$ does not necessarily imply a triplet pairing. Reliable data in $\left(\mathrm{TMTSF}_{2} \mathrm{ClO}_{4}[57\right.$ are not in contradiction with the picture of singlet pairing but no data exist below $0.5 \mathrm{~K}$, the temperature domain where it would be most rewarding to see how $H_{c 2}$ compares with the Pauli limit when $H$ is perfectly aligned along the $a$ axis. This new piece of information could select between singlet and triplet pairing hypothesis. The present study has also shown that the transport in the normal state cannot be explained by a conventional single particle Drude model. It supports the existence in the conductivity spectrum of a very narrow zero frequency peak carrying a minor fraction (about 1\%) of the total spectral 
weight as already inferred from the FIR spectroscopy data [5., 56, 59] and also emphazises the role of electron correlations in these low dimensional conductors.

N.Joo acknowledges the french-tunisian cooperation CMCU (project 01/F1303) and thanks S.Haddad, S.Charfi-Kaddour and M.Héritier for helpful discussions. We also acknowledge a useful discussion with N.Dupuis.

[1] D. Jérome, A. Mazaud, M. Ribault, and K. Bechgaard. J. Physique Lett., 41:L95, 1980.

[2] A. A. Abrikosov. Jour. Low Temp. Physics, 53:359, 1983.

[3] C. Bourbonnais and L. G. Caron. Europhys. Lett., 5:209, 1988.

[4] L. P. Gorkov and D. Jérome. J. Phys. Lett., 46:L643, 1985.

[5] I. J. Lee, M. J. Naughton, G. M. Danner, and P. M. Chaikin. Phys. Rev. Lett., 78:3555, 1997.

[6] I. J. Lee, S. E. Brown, W. G. Clark, M. J. Strouse, M. J. Naughton, W. Kang, and P. M. Chaikin. Phys. Rev. Lett., 88:017004-1, 2002.

[7] I. J. Lee, P. M. Chaikin, and M. J. Naughton. Phys. Rev. Lett., 88:207002, 2002.

[8] T. Vuletić, P. Auban-Senzier, C. Pasquier, S. Tomić, D. Jérome, M. Héritier, and K. Bechgaard. Eur. Phys. Jour. B, 25:319, 2002.

[9] K. Bechgaard, K. Carneiro, M. Olsen, F. B. Rasmussen, and C. S. Jacobsen. Phys. Rev. Lett., 46:852, 1981.

[10] M. Takigawa, H. Yasuoka, and G. Saito. J. Phys. Soc. Japan, 56:873, 1987.

[11] Y. Hasegawa and H. Fukuyama. J. Phys. Soc. Japan, $56: 877,1987$.

[12] S. Belin and K. Behnia. Phys. Rev. Lett., 79:2125, 1997.

[13] H. Mayaffre, P. Wzietek, D. Jérome, and S. Brazovskii. Phys. Rev. Lett., 75:4122, 1995.

[14] S. M. de Soto, C. P. Slichter, A. M. Kini, H. H. Wang, U. Geiser, and J. M. Williams. Phys. Rev. B, 52:10364, 1995.

[15] K. Kanoda, K. Miyagawa, A. Kawamoto, and Y. Nakazawa. Phys. Rev. B, 54:76, 1996.

[16] H.Elsinger, J.Wosnitza, S.Wanka, J.Hagel, D.Schweitzer, and W.Strunz. Phys.Rev.Lett., 84:6098, 2000.

[17] J. Müller, M.Lang, R.Helfrich, F.Steglich, and T.Sasaki. Phys.Rev.B., 65:140509, 02.

[18] S. Belin, K. Behnia, and A. Deluzet. Phys.Rev.Lett., 81:4728, 1998.

[19] M.Pinterić, S.Tomić, M.Prester, D.Drobać, O.Milat, K.Maki, D.Schweitzer, I.Heinen, and W.Strunz. Phys.Rev.B., 61:7033, 2000.

[20] M.Pinterić, S.Tomić, M.Prester, D.Drobać, and K.Maki. Phys.Rev.B., 66:174521, 2002.

[21] B.J.Powell and RH. McKenzie. Phys.Rev.B., 69:024519, 2004.

[22] P. W. Anderson. J. Phys. Chem. Solids, 11:26, 1959.

[23] A. P. Mackenzie, R. K. W. Haselwimmer, A. W. Tyler, G. G. Lonzarich, Y. Mori, S. Nishizaki, and Y. Maeno. Phys. Rev. Lett., 80:161, 1998.

[24] S. Bouffard, M. Ribault, R. Brusetti, D. Jérome, and K. Bechgaard. J. Phys. C, 15:2951, 1982.

[25] M. Y. Choi, P. M. Chaikin, S. Z. Huang, P. Haen, E. M.
Engler, and R. L. Greene. Phys. Rev. B, 25:6202, 1982.

[26] L. Zuppirolli. in Low Dimensional Conductors and Superconductors, D. Jérome and L. G. Caron editors, page 307. Plenum Press, New-York, 1987.

[27] M. Sanquer and S. Bouffard. Mol. Cryst. Liq. Cryst., 119:147, 1985.

[28] C. Coulon, P. Delhaes, J. Amiell, J. P. Monceau, J. M. Fabre, and L. Giral. J. Physique, 43:1721, 1982.

[29] I. Johannsen, K. Bechgaard, C. S. Jacobsen, G. Rindorf, N. Thorup, K. Mortensen, and D. Mailly. Mol. Cryst. Liq. Cryst., 119:277, 1985.

[30] K. Mortensen and E. M. Engler. Phys. Rev. B, 29:842, 1984.

[31] J. P. Pouget. in Low Dimensional Conductors and Superconductors, D. Jérome and L. G. Caron editors, page 17. Plenum Press, New-York, 1987.

[32] S. Tomić, D. Jérome, P. Monod, and K. Bechgaard. J. Physique, 44 C3:1083, 1983.

[33] P. Garoche, R. Brusetti, and K. Bechgaard. Phys. Rev. Lett., 49:1346, 1982.

[34] S.Tomić. PhD thesis, Université Paris Sud,Orsay, 1986.

[35] O. Traetteberg. Thesis. University Paris-Sud, 1993.

[36] S. Tomić, D. Jérome, D. Mailly, M. Ribault, and K. Bechgaard. J. Physique, 44 C3:1075, 1983.

[37] S. Ravy, R. Moret, J. P. Pouget, and R. Comes. Physica, 143 B:542, 1986.

[38] A long exposure time or a high current needed for achieving a good sensitivity resulted in the sample sublimation.

[39] We have checked that the inelastic scattering contribution behaving like $T^{2}$ in the temperature domain $10 \mathrm{~K}$ $20 \mathrm{~K}$ is not affected by the modification of the impurity scattering.

[40] V. Ilakovac, S. Ravy, K. Boubekeur, C. Lenoir, P. Batail, and J. P. Pouget. Phys. Rev. B, 56:13878, 1997.

[41] The phase diagram of the R-state of $\left(\mathrm{TMTSF}_{2}\left(\mathrm{ClO}_{4}\right)_{(1-x)}\left(\mathrm{ReO}_{4}\right)_{x}\right.$ reported in reference 36$]$ showing a slight decrease of $T_{A O}$ upon alloying has been obtained with a cooling rate five times faster than our smallest cooling rate.

[42] F.Beuneu and P.Monod. Phys. Rev. B, 18:2422, 1978.

[43] P. G. de Gennes. Superconductivity of Metals and Alloys. Addison-Wesley Publishing Company, 1989.

[44] The determination of the critical temperature in zero field from the extrapolation of the critical field could have been performed using any field orientation. The $c^{*}$ orientation has been chosen because it is the axis along which it is easier to orient the magnetic field.

[45] B. Korin-Hamzić, L. Forró, J. R. Cooper, and K. Bechgaard. Phys. Rev. B, 38:11177, 1988.

[46] M. Weger. J. Physique Coll., 39, C6:1456, 1978.

[47] J. Friedel and D. Jérome. Contemp. Phys., 23:583, 1982.

[48] J.Moser, M.Gabay, P.Auban-Senzier, D.Jérome, K.Bechgaard, and J.M.Fabre. Eur.Phys.J.B., 1:39, 1998.

[49] A.Georges, T.Giamarchi, and N.Sandler. Phys.Rev.B., 61:16393, 2000.

[50] P.Moses and R.McKenzie. Phys.Rev.B., 60:7998, 1999.

[51] J. R. Ferraro and J. M. Williams. in Introduction to Synthetic Electrical Conductors. Academic Press, London, 1987.

[52] K. Maki, H. Won, and S. Haas. Phys. Rev. B, 69:012502, 2004.

[53] A. I. Larkin. JETP Lett., 2:130, 1965.

[54] C. S. Jacobsen, D. B. Tanner, and K. Bechgaard. J. 
Physique, 44, C3:859, 1983.

[55] H. K. Ng, T. Timusk, and K. Bechgaard. Mol. Cryst. Liq. Cryst., 119:191, 1985.

[56] T. Timusk. in Low Dimensional Conductors and Superconductors, D. Jérome and L. G. Caron editors, page 275. Plenum Press, New-York, 1987.

[57] K. Murata, M. Tokumoto, H. Anzai, K.Kajimura, and
T. Ishiguro. Japanese Jour. of Appl. Physics, Suppl. 263:1367, 1987.

[58] F.Pesty, K.Wang, and P.Garoche. Synthetic. Metals., 27:137, 1988

[59] A. Schwartz, M. Dressel, G. Grüner, V. Vescoli, L. Degiorgi, and T. Giamarchi. Phys. Rev. B, 58:1261, 1998. 